\author{
Review of Computer Engineering Research \\ 2021 Vol. 8, No. 2, pp. 96-102. \\ $\operatorname{ISSN}(e): 2410-9142$ \\ $\operatorname{ISSN}(p): 2412-4281$ \\ DOI: $10.18488 /$ journal.76.2021.82.96.102 \\ (C) 2021 Conscientia Beam. All Rights Reserved. \\ check for \\ updates
}

\title{
A SMART SHELF DESIGN FOR RETAIL STORE REAL TIME INVENTORY MANAGEMENT AUTOMATION
}

\section{Ozgur TAMER ${ }^{1+}$ \\ Tunca KOKLU ${ }^{2}$}

\author{
Dokuz Eylul University, Turkey. \\ Email:ozgur.tamer@deu.edu.tr \\ -Aselsan Electronic A.S, Turkey. \\ Email:tunca.koklu@hotmail.com
}

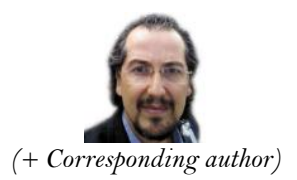

ABSTRACT
Article History

Received: 5 November 2021 Revised: 3 December 2021 Accepted: 14 December 2021 Published: 23 December 2021

Keywords

Real time inventory

Management

Smart shelf

Load cell.

\begin{abstract}
Conventional retail store inventory management systems rely on stockroom actions. However, especially in big scale retail stores, a certain amount of goods is placed on the display shelves. The items placed on the display shelves are not counted until their tags are identified by a cash register and marked as sold in the inventory management system. In this study, we propose a smart shelf that is capable of counting the specific items placed on it by detecting the location and the weight of the items. Our approach assumes that specific items in a retail store are placed in a specified location on each shelf, which is a widely preferred approach. The identified product information is then transferred to the inventory management system through the local network connection, and products on the display shelves can be counted in real time. The results show that the location and weight of the items can be identified with remarkable accuracy.
\end{abstract}

Contribution/Originality: This study is one of very few studies which have investigated a shelf design with load cells and wireless communication modules that enable real time inventory management of the items on the display shelves without using radio-frequency identification (RFID), QR codes or bar codes.

\section{INTRODUCTION}

In retail inventory management, it is essential to log products and record them as well as track changes due to sales or losses. A good retail inventory management system is capable of managing the flow of goods from the initial purchase to the final sale and provide the retailer with stock counts and necessary warnings. However, determining the location of every inventory item is complicated, especially for big retailers and retail chains. Items can be on display shelves or in the stockroom of a store. Most systems rely on the actions of the personnel responsible for managing stock, who should be able to present the number of items located on the display shelves and in the stockroom. This information may become unreliable if incorrect information has been entered into the tracking system by the authorized personnel. Therefore, it is essential to use technology to track the items placed on the display shelves as well as on the stockroom shelves.

Retailers employ automatic identification and data capture (AIDC) systems to acquire data of the products in their inventory. Two of the most preferred AIDC technology systems are printed code tags (bar codes and QR codes) and radio-frequency identification (RFID) tags. Bar code technology has been used in retail inventory management since the 1970s, and RFID gained prominence in the early 2000 s [1]. A case study to identify the impact of RFID on improving supply chain performance at two giant retail stores is presented by Omar \& Ray [2]. One retail store chain found that serving the promotional items on time and avoiding out-of-stocks achieved a $19 \%$ 
increase in sales. The other retail chain deployed second generation RFID tags to the point of sale (POS) cases. They also experienced that the improvement in merchandise availability influenced sales performance by $15 \%-20 \%$. The authors concluded that the use of RFID increased the flexibility of operations and reduced the cost of inventory management. An inventory management system based on RFID tags and Zigbee's wireless network for detecting the real-time location of the inventory items to reduce inaccuracies in inventory records has been proposed by Ong \& Kamsin [3]. The exact location of a particular item is also shown in the inventory management software based on the location of the shelves, columns and rows. The authors claim that the system is suitable for larger-scale logistics tracking and management applications. Lakshmi et al. proposed a system using QR codes and blockchain technology for transparent, distributed, and reliable inventory management [4]. The $\mathrm{P} 2 \mathrm{P}$ network of blockchain includes manufacturers, distribution centers, retail partners, suppliers and production sites as nodes. A QR code stores product details such as the ID of the product and the manufacturer, expiration date, cost and other relevant information. After the product has been purchased by the customer, a new block with updated details of that product is created and stored in the blockchain. Inventory can be evaluated by getting the products details from the blockchain database. Kalange et al. proposed a system for a store owner to maintain inventory information and place orders based on reading RFID tags and load cells [5]. A similar work presented by Singh et al. includes a weighing shelf for online grocery tracking [6]. In the presented work, the expenditure and the rate of exhaustion of the products is tracked using the data acquired from the load cells placed under the shelves. The system also employs RFID tags to identify the items placed in the sections of the shelf. The inventory can be tracked using an android app. A system based on a shopping cart that allows the automation of the shopping process has been presented by Shanmugavadivel \& Gomathy [7]. The system uses a camera and a pretrained neural network to recognize products that are placed in a cart or basket. A load cell is used to validate the weight of the products in the cart as a confirmation step. A similar system has been presented by Priya et al., which employs Raspberry Pi and RFID tags as well as load cells inside the shopping cart [8]. The RFID tags on the items are scanned before placing into the cart and the load cell compares the weights of the products and indicates if the correct item has been placed into the cart.

Manikandan presented a system that detects the weight of grocery items, identifies the items that need to be replenished, and generates an alert through a webpage [9]. The weight of each item is measured using a single load cell, while the shelf has four load cells and can measure the weights of four items simultaneously. In the work presented by Manuel et al., a smart food cabinet that can give information about the number of specific items stored via a mobile application and combine the data to display cooking recipes [10]. The system employs load cells to measure the weight of each item inside the cabinet.

A store management system to identify and monitor items placed on the shelves has been proposed by Jyothi \& Navya [11]. The system is capable of automatically alerting retailers for restocking. The system implements a load cell to detect products on the shelf. A shelf system, which can identify the number of items placed on it and track the number of products in stock in real time by using the data acquired from the load cells has been presented by Laszlo et al. [12]. The total product weight on the shelf is evaluated as the sum of the weights measured by the four load cells. According to the results presented in the paper, the system can be used to manage shelf inventory.

The main aim of inventory management systems is to count items in stock. The items placed on the display shelves are not counted until their tags are identified by a cash register and marked as sold in the inventory management system. This may result in an out-of-stock situation on a display shelf, especially for fast-selling promotional items. In this work, we propose a smart shelf equipped with four load cells capable of counting the number of items and detect their location. The main aim is to count specific items that are supposed to be placed on specific locations on the shelf, since in real life applications, a display shelf does not contain a single item but contains many different items side by side. With the help of the proposed system, an inventory management system 
will have the ability to not only count the items in the warehouse/stockroom but will also be capable of counting the items on the displays.

This paper is organized as follows: Section 2 defines the methodology and working principles of the hardware and the embedded software; Section 3 contains the results; and Section 4 concludes.

\section{METHODOLOGY}

The smart shelf proposed in this work measures the weight placed on load cells located on four sides of the shelf. A diagram presenting the locations of the load cells with respect to the shelf is presented in Figure 1. As presented in the figure, the shelf is placed on a rigid structure, while the shelf is placed on four load cells placed on four corners of the shelf. One end of the load cell is fixed to the shelf while the other end is fixed to the rigid carrier via standard bolts.

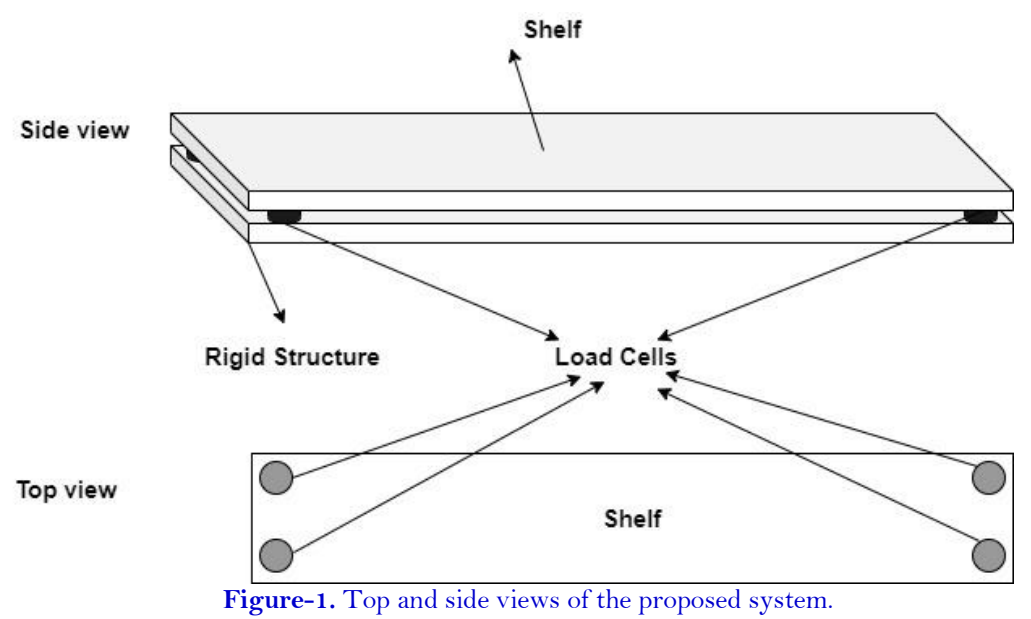

Weight measurement using multiple load cells is not a novel approach and it is well known that the total weight on the shelf is the sum of the measured weights by all of the load cells [13]. However, each of the load cells will record different weights depending on the position of the load [14]. In this study, we use this difference to estimate the location and weight of a product placed on the shelf. Schematic representation of the system is presented in Figure 2. Here, the weight of the load is measured by four beam load cells accompanied by an amplifier. The amplifier circuit includes a HX711 integrated circuit that includes an analog to digital converter and has a serial interface as the output for the converted data. The digital data is transmitted to a Raspberry Pi $3 \mathrm{~B}$ embedded computer which processes the information and estimates the weight and location of the load on the shelf.
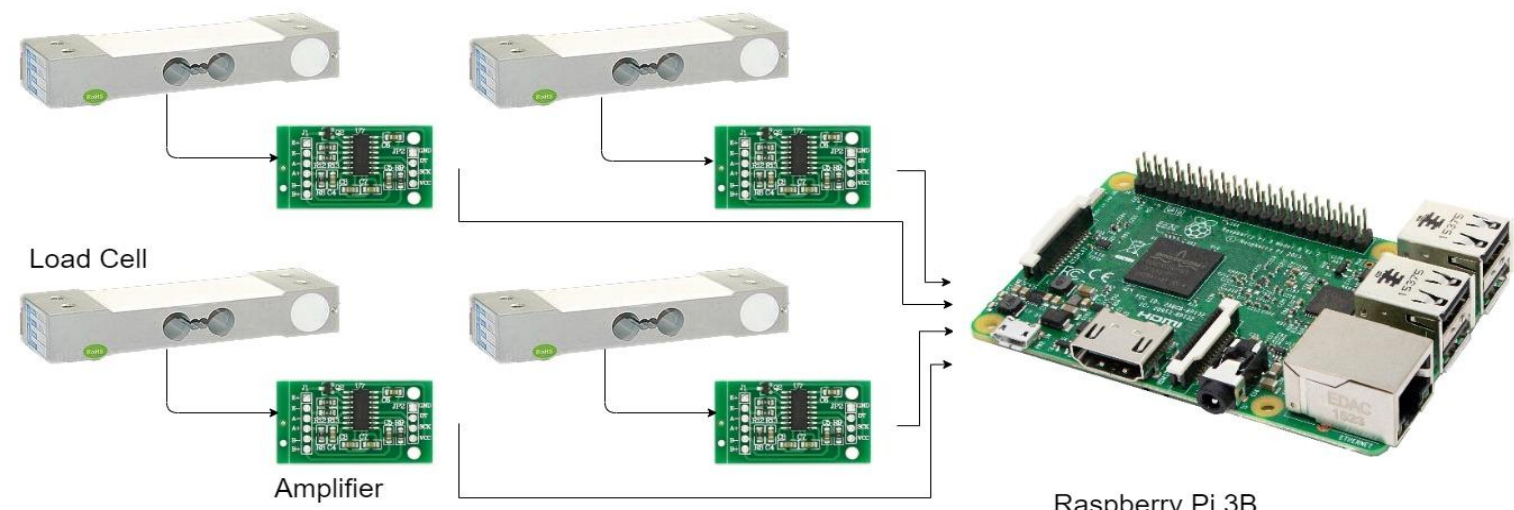

Raspberry $\mathrm{Pi} 3 \mathrm{~B}$

Figure-2. Schematic representation of the system. 
Even though identical load cells and amplifiers are employed in the system, there are still small differences due to imperfect mounting and mechanical defects on the load cells. These defects can be ignored when evaluating the overall weight; however, they can degrade the performance of the location estimation algorithm significantly. Therefore, a calibration procedure is necessary to compensate for these defects.

In order to calibrate the sensors, first the shelf is divided into four virtual regions, as presented in Figure 3, and an object with a weight below the maximum capacity of the load cells is placed on different regions of the shelf repeatedly. The calibration software acquires the weight on each load cell and asks the user the region of the load. It is expected that the load cell closest to the load will sense the largest impact due to placement/replacement of the load.

(a)

\begin{tabular}{|l|l|}
\hline 1 & 3 \\
\hline 2 & 4 \\
\hline
\end{tabular}

(b)

\begin{tabular}{|l|l|l|l|}
\hline 1 & 3 & 5 & 7 \\
\hline 2 & 4 & 6 & 8 \\
\hline
\end{tabular}

Figure-3. Regions of the shelf; (a) four regions; (b) eight regions.

After several tests, the software stores the calibration data as a reference and employs it during the location estimation process. The main software employs this calibration data and estimates the location of the load when placed on the shelf. The total weight of the load is also evaluated by adding up all of the load cell values and subtracting the weight of the shelf.

Each shelf in a retail store has a unique ID, and when the location and the weight of an item is successfully evaluated, the shelf ID and estimated location is transferred to the retail store's management software by the protocol presented in Table 1. Therefore, any software capable of reading this data format can integrate the proposed smart shelves in a retail store's management software.

Table-1. Data protocol format.

\begin{tabular}{c|c|c|c|c|c|c|c}
\hline Start & Date & Time & Shelf ID & Region ID & Placed/Replaced & Amount & End \\
\hline 1010 & $\mathrm{DD} / \mathrm{MM} / \mathrm{YY}$ & $\mathrm{HH} / \mathrm{MM}$ & $\mathrm{ID} \#$ & $1 / 2 / 3 / 4 \ldots$ & $1 / 0$ & $1-256$ & 0011 \\
\hline
\end{tabular}

\section{RESULTS}

A testbed has been built and is presented in Figure 4 The testbed shelf and the bottom part as the rigid layer are made from an aluminum sigma profile with built-in rails and screw holes to standardize the mounting of the load cells on all layers and to reduce mounting defects. The dimensions of the shelf are $1 \mathrm{~m}$ in width and $20 \mathrm{~cm}$ in depth, while the weight of the shelf is $6 \mathrm{~kg}$. Load cells, capable of sensing up to $5 \mathrm{~kg}$, are preferably located on each corner of the shelf, as presented in Figure 5. Mounting points marked in black are for the shelf, while points marked in white are for the bottom rigid layer.

In order to evaluate the performance of the testbed, test software has been developed. The interface of the test software is presented in Figure 6. As can be seen, there are four regions and one of them is marked in red. This means that the test weight has been placed here. The user then marks the correct region of the test weight from the check boxes below the regions and the test result is recorded accordingly. The upper button (BASLAT) is used to start the test procedure, and the lower button (CIKIS) is to exit the software. Calibration software with a similar 
interface was also developed and run prior to this test operation to create calibration data. The software is also capable of sensing eight fields instead of four fields, as shown in Figure 7.

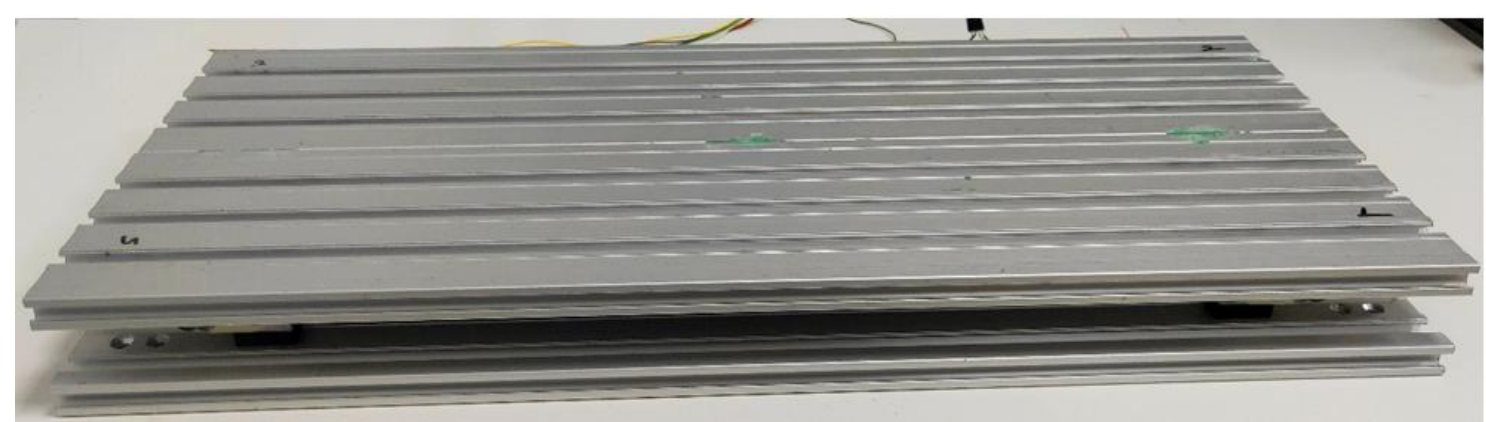

Figure-4. Testbed for demonstration of the proposed approach.

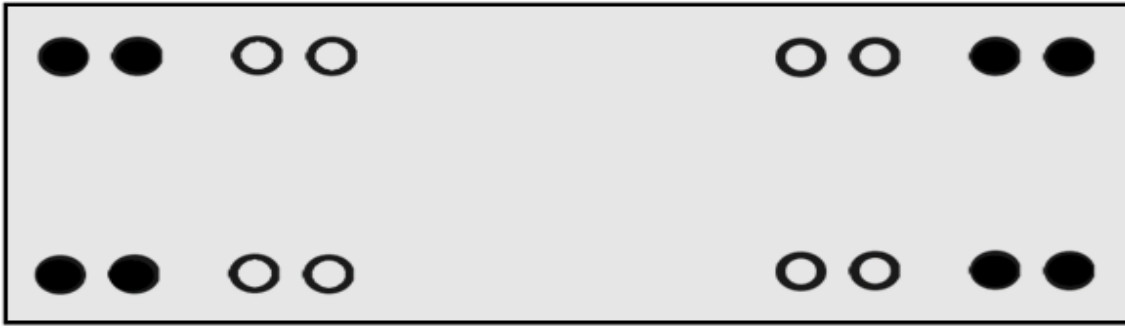

Figure-5. Mounting points for the load cells.

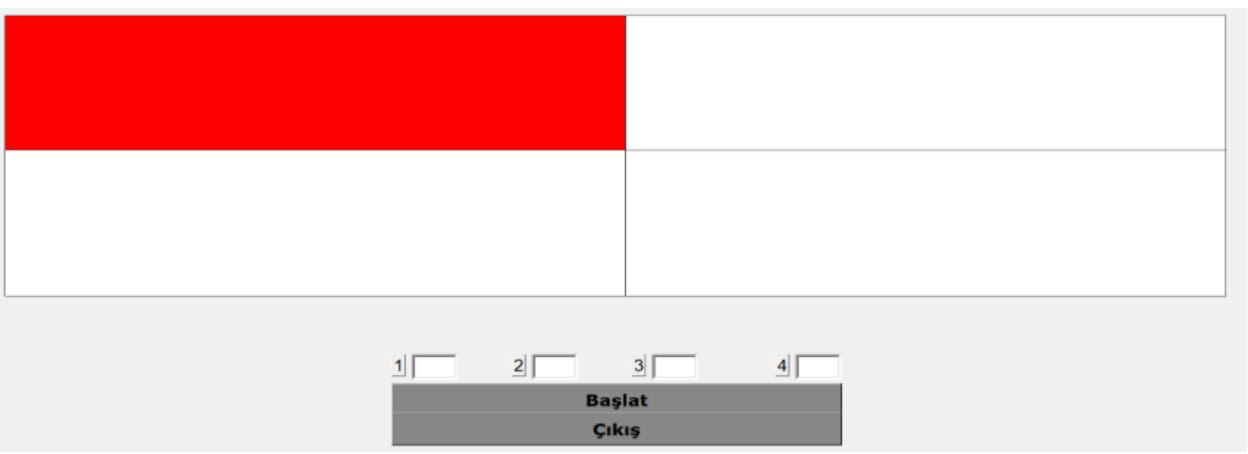

Figure-6. Test software interface.

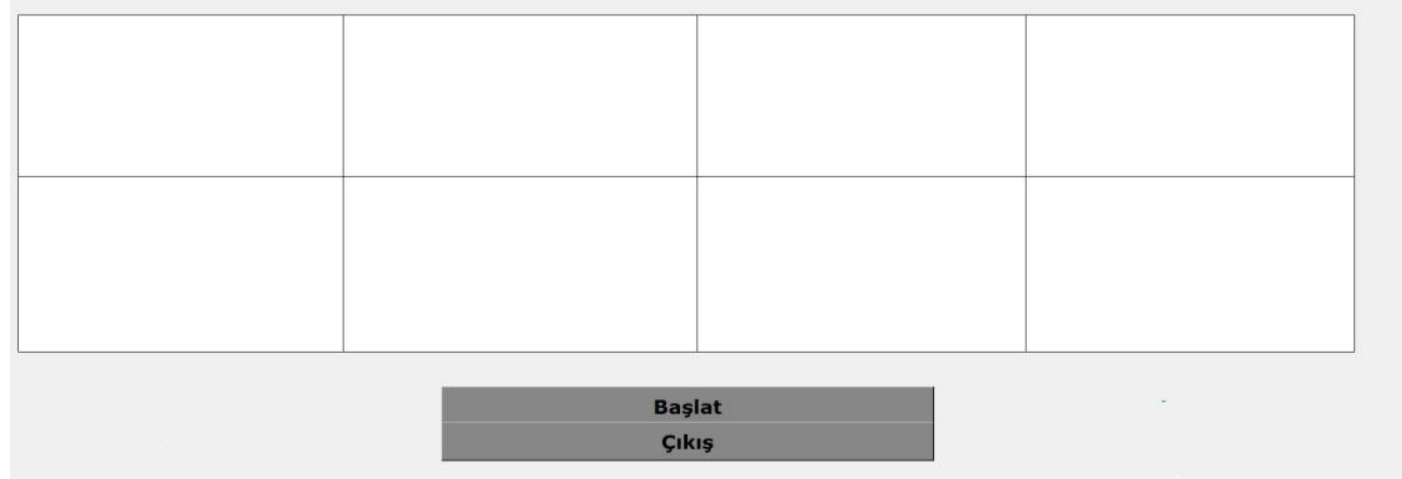

Figure-7. Eight-field functionality of the software.

By using the testbed and developed test software, several tests were performed using a $500 \mathrm{~g}$ weight to evaluate the performance of the proposed smart shelf. Before each test sequence, the shelf is calibrated using the software and the same calibration data is used throughout the test. The results of these tests are presented in Table 2. For the four-region test, two errors occurred. First, when the test load was in region 1, it was observed as region 4 , and second, when the test load was in region 3 it was observed as region 4. For the eight-region test, similar erroneous behavior occurred between regions 2 and 1 and regions 3 and 4 . 
Table-2. Test results

\begin{tabular}{c|c|c|c}
\hline Test & Number of Tests & Correct Estimates & Percentage \\
\hline Four-region Test & 80 & 78 & $97 \%$ \\
\hline Eight-region Test & 240 & 231 & $96 \%$ \\
\hline
\end{tabular}

\section{CONCLUSION}

A shelf mechanism, which can track the availability of the items placed on it, has been proposed in this paper. The shelf is equipped with load cells and a single board computer. The change of the weight on the shelf is sensed using the load cells and when a change occurs in a positive or negative direction (positive means an item has been placed while negative means an item has been picked), the region of the weight change is detected by the developed software and the change in the inventory on the shelf is transmitted to the store's inventory management software by the proposed data transfer protocol.

A testbed has been built to test the performance of the proposed method, which makes it very convenient to acquire the change in weight since the sensed weight change on all four load cells is summed up. When this change occurs, an estimation algorithm is run and the region of the weight change is estimated. The estimation algorithm performed with success rates of $96 \%$ for the eight-region shelf and $97 \%$ for the four-region shelf. It can be concluded that these error rates are more than satisfactory. Particularly, the measurement error was obtained when the weight of the load was close to the maximum value, which can be measured by the instrument. Therefore, it is essential to use load cells capable of measuring far beyond the maximum weight that can be loaded.

A data transfer protocol is also proposed in this paper, but the testbed did not include an ERP software interface, so it was not possible to test the performance of the system in a more realistic environment. However, this is planned as a future study. Another continuing study is increasing the regions using four load cells, which will need a more rigid mounting surface since transitions between the regions need precise measurements.

Funding: This work is supported by The Scientific and Technological Research Council of Turkey (TUBITAK) under grant number 7180360.

Competing Interests: The authors declare that they have no competing interests.

Acknowledgement: Both authors contributed equally to the conception and design of the study.

\section{REFERENCES}

[1] R. Atkins, A. Sener, and J. Russo, "A simulation for managing retail inventory flow using RFID and bar code technology," Decision Sciences Journal of Innovative Education, vol. 19, pp. 214-223, 2021.Available at: https://doi.org/10.1111/dsji.12232.

[2] A. Omar and H. Ray, "Improving the retailer industry performance through RFID Technology," pp. 196-220, 2018.

[3] G. Ong and I. F. B. Kamsin, "Implementation of zigbee-enabled rfid inventory management system in retail stores," PalArch's Journal of Archaeology of Egypt, vol. 17, pp. 8699-8708, 2020.

[4] G. Vidhya Lakshmi, S. Gogulamudi, B. Nagaeswari, and S. Reehana, "BlockChain based inventory management by QR code using open CV," presented at the In 2021 International Conference on Computer Communication and Informatics (ICCCI), IEEE, 2021.

[5] S. H. Kalange, D. A. Kadam, A. B. Mokal, and A. A. Patil, "Smart retailing using IOT," International Research Journal of Engineering and Technology (IRJET), vol. 4, pp. 263-268, 2017.

[6] S. Divyaansh, D. Neel, and R. N. Rohit, "Design of portable IoT based Grocery tracking system via Wi-Fi module for design of portable IoT based Grocery tracking system via Wi-Fi module for home automation," International Research Journal of Engineering and Technology (IRJET), vol. 7, pp. 5469-5473, 2020.

[7] G. Shanmugavadivel and B. Gomathy, "Smart shopping cart," International Journal of Advanced Science and Technology, vol. 28, pp. 91-97, 2019. 
[8] R. Priya, N. A. Swetha Shri, G. Krishnakumar, S. Kaliappan, and P. Jeyabharathi, "Electronic smart cart with billing assistance in super market," Iconic Research and Engineering Journals, vol. 3, pp. 202-204, 2020.

[9] T. Manikandan, D. T. Selvan, R. Vaidhyanathan, B. Vigneshvaran, and V. Nandalal, "Home groceries management system using IoT," International Journal Psychosocial Rehabil, vol. 24, pp. 2741-2746, 2020.

[10] C. Manuel, J. Avila, L. Budiman, R. Wijaya, and R. Hedwig, "Customizable smart food cabinet and refrigerator," Journal of Science \& Technology, vol. 27, pp. 143-157, 2019.

[11] G. D. Jyothi and K. Navya, "Design and implementation of a store management system," presented at the In 2017 International Conference on Intelligent Sustainable Systems (ICISS), IEEE, 2017.

[12] S. Laszlo, M. Laszlo, and D. Jozsef, "Intelligent IoT shelf design and development," presented at the In 2019 IEEE 19th International Symposium on Computational Intelligence and Informatics and 7th IEEE International Conference on Recent Achievements in Mechatronics, Automation, Computer Sciences and Robotics (CINTI-MACRo), IEEE., 2019.

[13] R. Lolley, "A review of industrial weighing systems (Part 2)," Measurement and Control, vol. 9, pp. 435-439, 1976.

[14] K. Thakkar, M. P. Dhaval, and A. P. Narendra, "Finite element analysis of single point beam type load cell to optimize it's measuring range," presented at the Recent Advances In Manufacturing (Ram2010), National Institute of Technology, Surat, 2010, 2010. 